\title{
EVALUACIÓN DE UN MODELO DE GESTIÓN DE INNOVACIÓN EN LA PRÁCTICA EDUCATIVAAPOYADA EN LAS TIC. ESTUDIO DE CASO: UNIMET.
}

\section{EVALUATION OF A MODEL OF INNOVATION MANAGEMENT IN THE ICT-BASED EDUCATIONAL PRACTICE. CASE STUDY: UNIMET}

\author{
Dra. Ivonne Candissi Harvey López \\ iharvey@unimet.edu.ve
}

Universidad Metropolitana. Facultad de Ciencias y Artes.

Departamento de Ciencias de la Educación.

Distribuidor Universidad Autopista Petare-Guarenas. Urbanización Terrazas del Ávila.76819, Caracas (Venezuela)

La Universidad Metropolitana ha sido una organización educativa que ha desarrollado experiencias de incorporación e innovación tecnológica; la investigación brindó una evaluación general de su Gestión de la Innovación. La investigación fue de carácter cualitativo, evaluativa, con Estudio de Caso, de tipo intrínseco e instrumental considerando el estudio en profundidad. La Unimet ha venido planificando sus procesos organizando sus recursos para permitir y facilitar la innovación en la producción y difusión del conocimiento y en los procesos de enseñanza y aprendizaje, sin embargo aún se deben mejorar algunos procesos para optimizar lo académico y administrativo y consolidar el trabajo.

Palabras clave: Gestión de la innovación, práctica educativa en Educación Superior, Tecnologías de la Información y la Comunicación, evaluación institucional, Universidad Metropolitana.

Universidad Metropolitana (Unimet) has been an educational organization that has developed experiences in incorporation of technological innovation; this research provided a general assessment of its management of innovation.

We did in-depth Case Study research with qualitative and evaluative nature, intrinsic and instrumental type. The Unimet has been planning its processes, organizing and resources to allow innovation in production, dissemination of knowledge, teaching and learning processes, however, it should improve some processes to optimize academic and administrative performance, also consolidate the team work capabilities.

Keywords: Innovation management, educational practices in higher education, ICT, institutional assessment, Universidad Metropolitana. 


\section{Introducción.}

El uso de las Tecnologías de la Información y la Comunicación (TIC) ha propiciado el desarrollo de nuevos escenarios de acción, donde la información y el conocimiento juegan un papel fundamental, debido a la rapidez con que es difundida la información, su accesibilidad y a la necesidad de transformarla en conocimiento para el desarrollo de nuevas propuestas tecnológicas.

Para lo educativo, estos escenarios han significado un redimensionamiento de la estructura, funcionamiento y acción de las organizaciones escolares y muy particularmente una revisión y modificación de los roles de los actores involucrados. Históricamente las TIC han venido y vienen acompañadas de un conjunto de cambios que obligan de una manera u otra a revisar el hacer educativo, sea por decisión de particulares o porque la misma dinámica obliga a repensar y a generar líneas de acción para sistematizar las experiencias e institucionalizarlas.

Las instituciones de Educación Superior han intentado buscar alternativas de incorporación de las TIC en la práctica educativa, y se han generado todo un conjunto de experiencias que reflejan, formas de implementación y desarrollo que responden a una manera de hacer particular que no es más que un proceso de gestión. La gestión para la presente investigación representó un aspecto relevante, considerado que en la actualidad las universidades se enfrentan a los retos que la sociedad de la información y del conocimiento le imponen, y que se asumen como organizaciones socialmente activas, interconectadas y que mantienen su función de ser productoras y usuarias de conocimiento para el desarrollo; siendo así las TIC dinamizan aún más esta función, por lo que la innovación representa el ingrediente principal para motorizar los cambios, asumiendo que se requiere ofrecer respuestas acertadas y pertinentes a las actuales demandas.

La Universidad Metropolitana en Venezuela, se ha caracterizado por ser una organización educativa que durante casi 4 décadas ha desarrollado experiencias de incorporación e innovación tecnológica. Al ser una institución cuya finalidad es la formación de profesionales en diversas áreas del conocimiento, ha tenido una orientación particular en lo que al uso de tecnología se refiere, porque ha asumido como parte de su contexto de formación el hecho de que las Tecnologías de la Información y la Comunicación (TIC) han generado una reformulación de la práctica educativa al contar con nuevas herramientas que facilitan procesos de comunicación y acceso a la información, por lo tanto se ha asumido la tecnología desde una perspectiva pedagógica, desde sus posibilidades de uso en los procesos formativos.

Ahora bien, de acuerdo a lo expresado por La Guardia (2004, p.2) toda esta discusión en el ámbito educativo tiene sus particularidades, en la Educación Superior, hoy día el rol de las universidades ha cambiado, la concepción tradicional que se venía manejando de «...organización social, en la cual se forman individuos portadores de un conjunto de conocimientos que los califican para el ejercicio profesional y la vida en sociedad»; y se transforma hacia lo que la UNESCO ha llamado en su Informe: la Universidad del Siglo XXI según García (2001): 
Una organización socialmente activa, abierta e interconectada con su entorno y en la cual se formen individuos portadores de una cultura de aprendizaje continuo, capaces de actuar en ambientes intensivos en información, mediante un uso racional de las nuevas tecnologías de la información y las comunicaciones (p.2).

Asumiendo esta concepción, las universidades venezolanas y particularmente la Universidad Metropolitana ha desarrollado experiencias de innovación, asociadas a la incorporación de las TIC, donde la innovación, representa mucho más que experiencias aisladas y de procesos administrativos para el cambio dentro de una organización educativa, es lo que en palabras de Escudero (2003) se concibe como un proceso de definición, de construcción y de participación social, donde se desarrolla una búsqueda por un ideal compartido para capacitar y potenciar las instituciones en pro de los retos y exigencias a los que frecuentemente está expuesta.

Ello implica que el concepto de innovación, como lo hemos señalado anteriormente para que tenga sentido, requiere ser parte de la gestión, ya que tradicionalmente los actores institucionalmente desarrollan procesos de innovación lo que se requiere es que la gestión favorezca estos procesos y se logre el desarrollo de experiencias de innovación permanentes. Gestión e innovación vistas en conjunto conforman o están soportadas en una visión educativa dinámica, cambiante, donde la información y el conocimiento son ejes fundamentales; razón por la cual se pretendió a través de esta investigación vincular estos elementos a través de lo que algunas organizaciones han llamado Gestión de la Innovación, que se asume como:

«...conjunto de acciones que permiten planificar, desarrollar y evaluar los procesos educativos para lograr la innovación; considerando para ello tres elementos

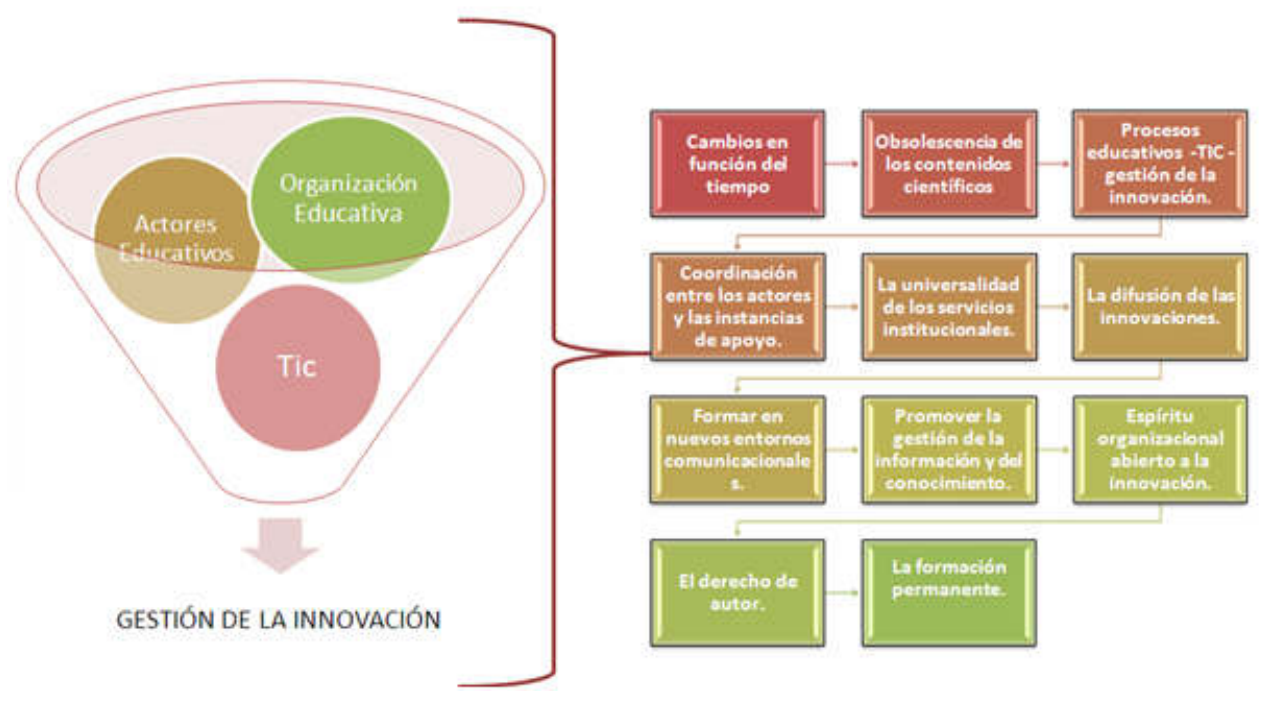

Figura 1. Modelo de Gestión de Innovación (Harvey, 2014).

Píxel-Bit. Revista de Medios y Educación. No 47 Julio 2015. ISSN: 1133-8482. e-ISSN: 2171-7966. doi: http://dx.doi.org/10.12795/pixelbit.2015.i47.09 
fundamentales: La Organización Educativa, los actores educativos (comunidad educativa en general), y las Tecnologías.» Cada uno de estos elementos se conjugan para alcanzar, lo que se asume como una de las finalidades de la Universidad en la actualidad: la generación y difusión de conocimientos a través de la investigación; la certificación profesional; y la innovación de los procesos de enseñanza y aprendizaje que se evidencian en la docencia y la extensión (Harvey, 2010, p. 243).

Bajo este escenario, se desarrolló un modelo de gestión de innovación para proyectos de incorporación de TIC (Ver Figura 1), el cual se implementó dentro del contexto de la Universidad Metropolitana, para ser evaluado considerando como criterios: la organización educativa, los actores educativos y las tecnologías con las que cuenta la universidad para llevar a cabo prácticas de incorporación de TIC en los procesos educativos.

Al revisar el contexto nacional e internacional, tanto a nivel de investigaciones como de desarrollo de políticas educativas nos encontramos con realidades muy diversas, y desde esta perspectiva se han venido desarrollando un conjunto de políticas públicas (Ver Figura 2) relacionadas con la incorporación de las TIC en diversos escenarios, y resultan importantes destacar ya que representan el contexto en el que se ha desenvuelto la Universidad para cumplir con parte de su visión institucional.

Es importante destacar, que la creación y promoción de cada una de las propuestas académicas de la universidad fueron propuestas innovadoras, que en su momento dieron respuesta a las demandas no sólo

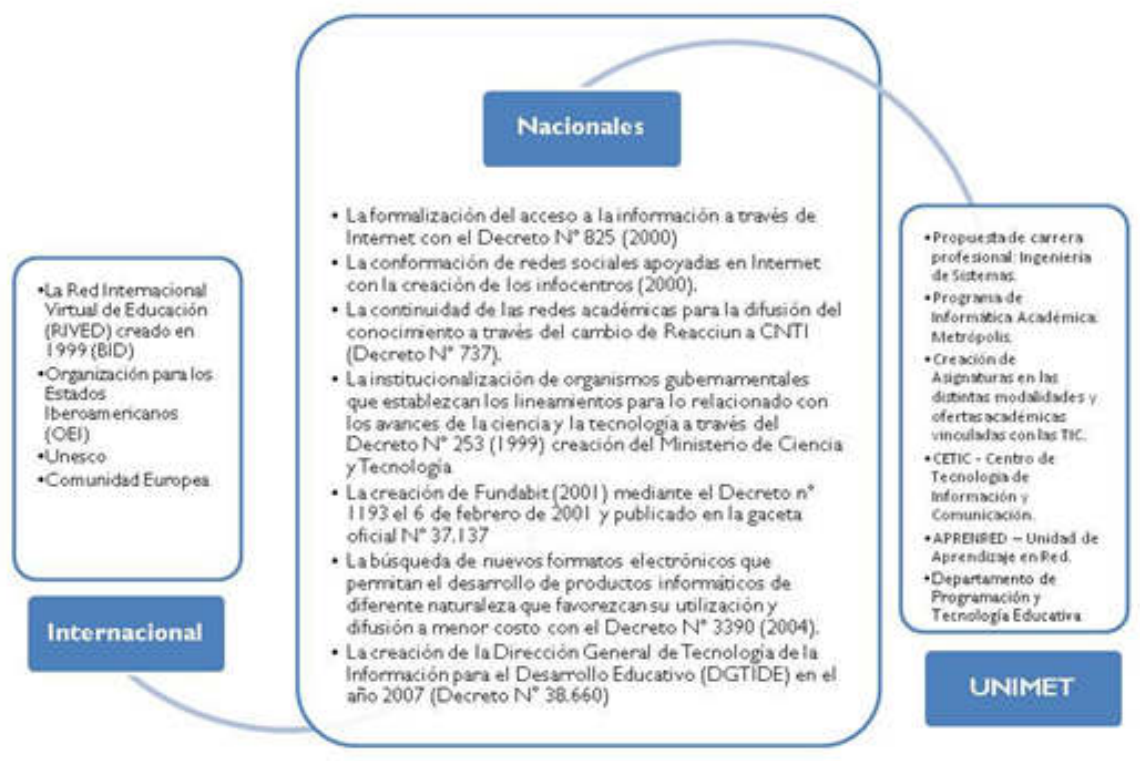

Figura 2. Políticas públicas vinculadas con las TIC internacionales y nacionales (Harvey, 2014). 
Innovación dentro de las universidades y en los actuales contextos, nos ubica en un plano de reflexión constante, (...) va más allả de una implementación de políticas y circunstancias externas, representa un reto permanente (...). Con la incorporación de las TIC en los procesos educativos. los escenarios de enseñanza y aprendizaje, toman un nuevo rumbo, pero no sólo por lo que implica a nivel de desarrollo de equipos tecnológicos y de inversiones para crear y mantener una infraestructura, sino por lo que significa para los actores involucrados que son los que en definitiva llevan a cabo el cambio y la mejora

\section{Características}

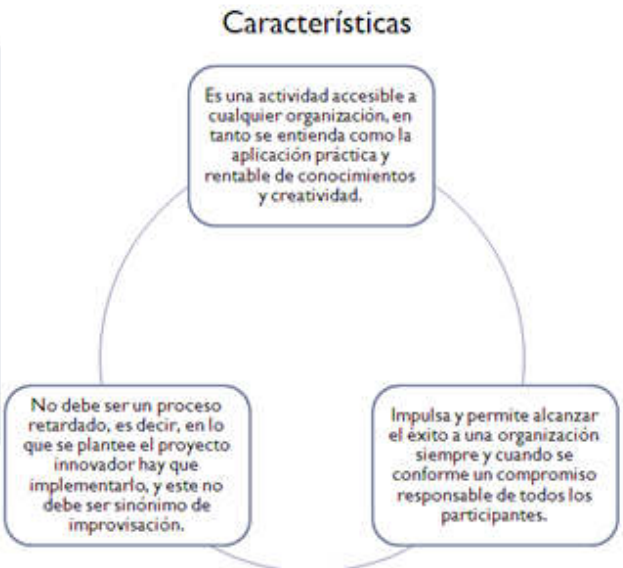

Figura 3.Definiciónde Innovación y característica. (Harvey, 2014).

\section{Caracteristicas}

Gestión educativa que fomente las bases
políticas, los lineamientos, normativas,
reglamentos, etc., que favorezcan procesos
innovadores dentro de una institución; pero
además es importante que en niveles más
específicos la gestión escolar cuente con un
personal calificado, comprometido.y que
tenga además los recursos y la infraestructura
para llevar a cabo estas prácticas educativas.
donde la innovación sea un proceso
permanente. Realidad que lamentablemente no
siempre se cumple en el sistema educativo
venezolano, pero que sin embargo no representa
un obstáculo para que se generen experiencias
novedosas

Gestión educativa que fomente las bases reglamentos, etc., que favorezcan procesos innovadores dentro de una institución; pero además es importante que en niveles más tenga además los recursos y la infraestructura para llevar a cabo estas prácticas educativas. donde la innovación sea un proceso permanente. Realidad que lamentablemente no venezolano, pero que sin embargo no representa novedosas

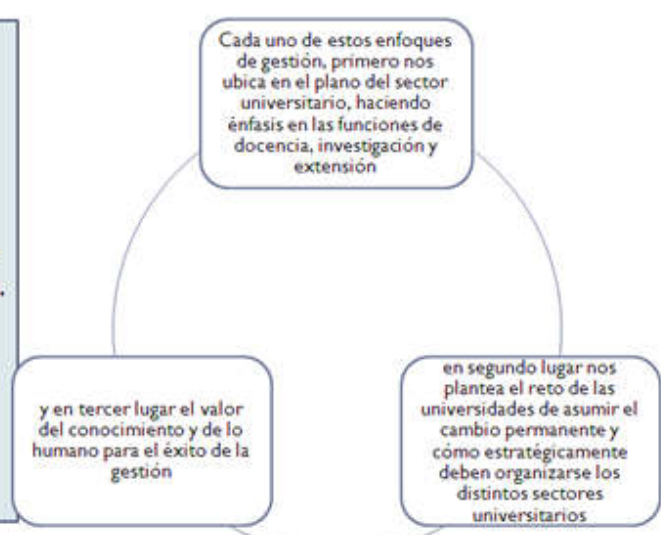

Figura 4.Definición de Gestión y características (Harvey, 2014).

nacionales sino internacionales que se estaban gestando en el mundo (Castell, 2002; Delors, 1996; Morín, 2000) y que requerían de organizaciones educativas capaces de formar profesionales para sociedades cambiantes, complejas, llenas de incertidumbres y con competencias para una formación para toda la vida (Cabero et al., 2002; Cabero \& Llorente, 2006; García, 2012).

Para lo educativo, estos cambios no han pasado desapercibido, debido a que surgen nuevas realidades, nuevos ciudadanos que 
requieren formación, nuevas profesiones, nuevos contenidos para enseñar y nuevas estrategias para hacerlo, ello ha implicado la necesidad de revisar la finalidad de la educación y generar nuevas maneras de hacerla apoyada en el uso de nuevas herramientas tecnológicas (Barroso, 2007; Cabero, 2006; Castaño, 2009; Rodríguez, 2009).

Siendo así, los procesos educativos y formativos en la educación superior particularmente, toman nuevos rumbos (Cabero, 2005; Esteve, 2009; García, 2012; Organización Internacional del Trabajo - OIT, 1998; Silvio, 2000), ya que si consideramos lo señalado anteriormente, las organizaciones escolares tradicionales (tal y como las conocemos) ya no representan los únicos espacios donde poder generar procesos de enseñanza y aprendizaje.

Ante toda esta realidad de cambios las estructuras tradicionalmente concebidas para la formación de los individuos requieren considerar este contexto, revisarse a sí mismas y actuar en pro de su propio proceso de cambio. Deben atender a los sujetos que ahora poseen nuevos modos de aprender teniendo la potestad de decidir el qué, el cómo y el cuándo; y para ello se debe contar entre otras alternativas, con organizaciones capaces de gestionar nuevos entornos telemáticos para atender estas necesidades (García, 2012; Morales, Medina \& Alvarez, 2003; Salinas, 2004; Unesco, 2005).

Desde esta perspectiva, la innovación (Ver Figura 3) se ha convertido en una dimensión clave para comprender la dinámica de las universidades y particularmente de la Universidad Metropolitana (UNIMET), porque representa el proceso mediante el cual los actores educativos han incorporado nuevas experiencias y nuevas herramientas a los procesos de docencia, investigación y extensión, además de los procesos académicos - administrativos que implica una universidad, cuyas características (carreras, modalidades, cantidad de alumnos, entre otros) hacen que el proceso de análisis de la innovación sea complejo y diverso.

Ahora bien, cuando se hace referencia a la gestión, no es más que acciones organizadas para lograr objetivos propuestos; la Universidad en general y particularmente la Universidad Metropolitana, ha asumido como parte de sus retos generar innovaciones apoyadas en el uso de las tecnologías, y todo ello requiere de una infraestructura capaz de generar las políticas y lineamientos para que se materialice (De Pablos, 2012; Rodríguez, 2012). Desde esta visión, se puede resumir que para la presente investigación se asume la gestión de acuerdo a la Figura 4.

El Modelo de Gestión de la Innovación (Harvey, 2010) propuesto en la presente investigación se orienta a tres grandes dimensiones:

a) La organización educativa (que para la presente investigación fue la Universidad Metropolitana) debe brindar un escenario que considere lo siguiente:

Desarrollo de políticas, lineamientos y/o normativas que estén en consonancia no sólo con las finalidades de la Universidad, sino que también respondan al contexto social que las rodea.

- Desarrollo de planes de difusión y de promoción de las políticas y acciones llevadas a cabo por la universidad y sus distintas instancias, para que toda la comunidad educativa esté informada y se sienta partícipe de los cambios generados dentro de la misma.

Mantener y fortalecer una política de formación permanente que favorezca la 
vinculación de los actores educativos no sólo con las tecnologías de la información y la comunicación (TIC) y sus usos educativos, sino también con la misión y visión de la universidad y su rol dentro del contexto social.

Brindar el soporte tecnológico mínimo necesario (redes, conexión, equipos, soporte permanente, entre otras) para el desarrollo de experiencias de incorporación de TIC tanto en la práctica educativa de sus docentes, en los procesos académicos y administrativos de las distintas instancias de la universidad, y en los procesos comunicacionales dentro de la misma.

b) Los actores educativos (que para la presente investigación son los profesores, estudiantes y el Equipo Rectoral) los cuales deben generar acciones que consideren lo siguiente:

Creación y desarrollo de proyectos permanentes que favorezcan prácticas educativas innovadoras, que incorporen las TIC en las distintas modalidades y propuestas educativas que mantiene la Universidad Metropolitana.

Desarrollo de experiencias de enseñanza y aprendizaje permanentes, que favorezcan la innovación, el desarrollo de nuevas prácticas, estrategias, metodologías y recursos haciendo uso de las TIC.

c) Las tecnologías (que para la presente investigación representa el soporte tecnológico-administrativo que brinda la Universidad Metropolitana) deben considerar lo siguiente:

Fortalecer la dotación de equipos de manera permanente, apoyándose para ello en el desarrollo de proyectos (individuales y grupales) que permitan obtener financiamiento para la Universidad.
Fortalecer las acciones entre las distintas instancias universitarias para mejorar los problemas de conexión a internet.

Favorecer el apoyo permanente a los procesos académico-administrativos.

\section{Metodología.}

Se asumió una investigación que intentó evaluar y vincular un Modelo de Gestión de la Innovación de la práctica Educativa apoyada en las TIC con una Organización Educativa: La Universidad Metropolitana, para ello se plantearon varios momentos:

a) Momento 1 - Diagnóstico Profesores: revisión de la práctica educativa de los profesores vinculadas con la implementación de las TIC en los procesos de enseñanza y aprendizaje; lo que implicó determinar las opiniones de los docentes en sus actividades profesionales. Para el momento de cierre de la investigación (20132014), el número total de profesores fue de 475. De los cuales 141 (29,7\%) eran profesores a tiempo completo y $334(70,3 \%)$ eran profesores a tiempo parcial; el número total de profesores que contestaron la encuesta fue de 109 profesores que representa aproximadamente el $23 \%$.

b) Momento 2 - Diagnóstico alumnos: revisión de las opiniones de los estudiantes en cuanto a su percepción de cómo sus profesores llevan a cabo sus prácticas educativas con el uso de las TIC, y cómo perciben ellos a la Universidad Metropolitana en cuanto a la Gestión que brinda para dar soporte a estas prácticas y a los procesos académico-administrativos que se dan en una Organización Educativa en Educación Superior. De acuerdo a datos suministrados por el Decanato de Estudiantes y por Control de Estudios la población total de estudiantes 
de la Universidad Metropolitana para el período 2013-2014 fue de 4.049. Para el cierre del proceso se logró contar con la opinión de 580 estudiantes que representa aproximadamente el $15 \%$.

c) Momento 3 - Diagnóstico Equipo Rectoral e informantes claves: entrevista a todo el personal que conforma el equipo rectoral de la Universidad Metropolitana para conocer sus visiones, permitió establecer un punto de partida para la reflexión, y el establecimiento de relaciones entre las distintas dimensiones (alumnos, profesores, teoría, políticas, etc.); y resulta importante destacar que se contó con la participación de informantes claves, que fueron seleccionados considerando los siguientes criterios: a) vinculación con el desarrollo de propuestas de incorporación tecnológica dentro de la Universidad; b) vinculación con procesos de gestión e innovación dentro de la organización educativa.

d) Momento 4-Diagnóstico de la Infraestructura Tecnológica: análisis de los recursos disponibles apuntando hacia las siguientes dimensiones: ¿Qué estrategias tecnológicas se están desarrollando?, ¿Dónde está el activo tecnológico y cuáles son sus características para desarrollar proyectos vinculados a la práctica educativa?, y ¿Cuáles son las tendencias y las previsiones que pueden construirse considerando el parque tecnológico existente?

e) Momento 5 - Revisión de los procesos de instruccionales y su gestión: análisis de contenidos de documentos, los cuales están vinculados a las políticas institucionales de incorporación tecnológica, innovación y gestión tanto de la Universidad Metropolitana, como del contexto macro universitario y el de otras universidades y organizaciones.
Para la presente investigación, partimos de dos premisas: la primera que es un estudio de carácter cualitativo y la segunda que tiene una naturaleza evaluativa.

De acuerdo a Strauss y Corbin (1990, citado en Sandín, 2003) establece lo siguiente:

«...por investigación cualitativa entendemos cualquier tipo de investigación que produce resultados a los que no se ha llegado por procedimientos estadísticos $\mathrm{u}$ otro tipo de cuantificación. Puede referirse a investigaciones acerca de la vida de las personas, historias, comportamientos, y también al funcionamiento organizativo, movimientos sociales o relaciones e interacciones. Algunos de los datos pueden ser cuantificados pero el análisis en sí mismo es cualitativo» (p. 121).

La naturaleza de la presente investigación estuvo dirigida a hacer una evaluación de la organización y las opiniones de sus actores; nos ubicó en un contexto donde los discursos y las acciones fueron fundamentales para recabar información pertinente. En este sentido, desde la visión de la investigación evaluativa, es importante comprender que entendemos por evaluación, y cómo este proceso representa el punto neurálgico de la investigación, para ello asumimos el concepto elaborado por Camperos (2012): «La evaluación requiere contar con un modelo deseable que represente el ideal de lo que se quiere evaluar; en otras palabras, la evaluación es una investigación totalmente orientada, dirigida por un marco de lo que debería ser o se espera de la situación o ente que se está examinando...» (p. 3).

Se usaron las siguientes técnicas de recolección de información:

1) La observación por encuesta y entrevista: la cual se apoya en instrumentos como los cuestionarios, los guiones de 
entrevista, acompañadas de aparatos de grabación y filmación.

2) El análisis de documentos: que se apoya en instrumentos auxiliares para registrar y analizar la información extraída de los documentos, que para la presente investigación se hizo a través de matrices de análisis y categorías establecidas por el propio modelo de Gestión de la Innovación.

De acuerdo a la revisión teórica llevada a cabo, la entrevista es asumida como una reunión para conversar e intercambiar información entre una persona (el entrevistador) y otra (el entrevistado) u otras (entrevistados).

Otra de las técnicas de recolección de información utilizadas fue el cuestionario entendido como: «...un instrumento de recolección de datos, integrado por preguntas que solicitan información acerca de un problema, objeto o tema de investigación, el cual es normalmente administrado a un grupo de personas» (Ruiz, 2002, citado en Navas, 2007, p.155).

Los instrumentos utilizados fueron ad hoc, y dependiendo de los sujetos participantes de la investigación, el cuestionario y las entrevistas tuvieron algunas diferencias; dicha decisión se sustentó en que cada población tiene características particulares académicas, profesionales y personales; sin embargo, en la Tabla 1 se puede observar el sistema categorial utilizado para los tres instrumentos.

De acuerdo a Taylor y Bogdan (1984, 1990, citado en Díaz, 2006) «Todos los investigadores desarrollan sus propios modos de analizar los datos cualitativos» (p. 159); basándonos en esta idea, es importante señalar que también se utilizó como base para el análisis de los datos la Teoría
Fundamentada de Glaser y Strauss (1967) la cual:

... se basa en una guía sistemática y flexible para la recogida y el análisis de datos cualitativos a fin de construir una teoría basada (o grounded) en los mismos datos. De este modo, los datos forman los cimientos de la teoría y su análisis genera los conceptos o categorías teóricas sobre los que se construye, ya que va más allá de la mera descripción de las respuestas al interpretar y explicar lo que ha sucedido (p. 3).

Esta teoría permitió la comprensión del contexto de la Universidad Metropolitana a través de sus procesos comunicacionales, que fueron posibles registrar gracias a los resultados obtenidos de los cuestionarios y de las entrevistas llevadas a cabo con los sujetos participantes de la investigación.

\section{Resultados.}

En este apartado se expone de manera resumida y general, los resultados del análisis de los datos provenientes de los cuestionarios aplicados a docentes y a estudiantes del ámbito de investigación y, la información proveniente de las entrevistas en profundidad hecha a los informantes concebidos como clave en el estudio.

Entre un $75 \%$ y $85 \%$ de la población objeto de estudio, tiene un conocimiento amplio de la existencia de herramientas tecnológicas para la enseñanza y para el aprendizaje y coinciden en una mayoría casi absoluta en dichas herramientas.

- De acuerdo a los resultados obtenidos aproximadamente un $70 \%$ de los estudiantes usan con mayor frecuencia las herramientas tecnológicas para gestionar su aprendizaje, que los profesores para gestionar sus procesos de enseñanza. Sin embargo, desde 
PROPÓSITO DEL INSTRUMENTO

1) Diagnosticar los elementos que favorecen un proceso de gestión de la innovación con el uso de las TICen los actores educativos de la Unimet.

2) Analizar los procesos de gestión de experiencias universitarias dirigidas a favorecer una práctica educativa que incorpore las TIC en la organización educativa en la Unimet.
DIMENSIONES SIGNIFICADO

Datos generales

\section{Gestión} Experiencias Universitarias con TIC

Permite evidenciar información general acerca de los Sujetos participantes de la investigación. (Profesores de la Unimet)

Permite

determinar el nivel de conocimiento del Informante acerca de su propia percepción, del manejo de las TIC en sus de prácticas educativas; $y$ cómo se han venido gestionando las experiencias educativas incorporando TIC en las distintas instancias académico administrativas de la Unimet Permite determinar el nivel de reflexión del informante acerca de cómo

Innovación en la práctica educativa lleva a cabo sus prácticas

educativas incorporando TIC; y su visión acerca de la formación de profesionales dentro de la Institución.

\section{INDICADORES}

- Género

- Grado instrucción

- Edad

- Tiempo Dedicación a la Institución

- Figura académicoadministrativa

- Ubicación Administrativa en la Institución

- Conocimiento acerca de las herramientas tecnológicas.

- Frecuencia de uso de las TIC.

- Nivel experticia del uso de las herramientas tecnológicas.

- Procesos gestión institucional para implementar las TIC en las instancias académico administrativas de la Unimet.
TIPO DE ÍTEMS

Respuesta abierta $y$ selección
Respuesta abierta y selección
- Nuevas prácticas educativas con la incorporación de las TIC.

- Visión acerca de la formación de profesionales en la Unimet.
Respuestas abiertas

Tabla 1. Sistema Categorial de los instrumentos de recolección de información (Harvey, 2014). 
la visión de los informantes claves y equipo rectoral mantienen una posición institucional de que se han llevado a cabo esfuerzos para que toda la comunidad esté vinculada a las bondades que ofrecen las tecnologías en las prácticas educativas.

A pesar de existir diversas herramientas tecnológicas adquiridas por la Universidad tanto los docentes como los estudiantes reflejan no tener una frecuencia de uso de las mismas; mientras la mayoría de los docentes dice haberla usado alguna vez, en los estudiantes predomina la idea de nunca haberlo hecho.

Desde la visión del estudiante la Universidad Metropolitana mantiene en su política universitaria el uso y gestión de las TIC para la enseñanza, posee la plataforma para hacerlo pero los profesores requieren de mayor adiestramiento en el uso de dichas TIC como apoyo a sus labores docentes.

En cuanto al reconocimiento, uso y experticia de las herramientas para el diseño de recursos de aprendizaje los docentes manifestaron de manera predominante su desconocimiento y los estudiantes, aunque se consideraron en un nivel de experticia básico, dijeron no haber usado dicha herramienta durante prácticas de sus docentes.

Aunque la Universidad tiene una plataforma educativa propia (Platum), tanto los docentes como los estudiantes no están sacando provecho de ésta pues, según los datos obtenidos, están haciendo mayor uso de páginas Web para la administración de las asignaturas.

\section{Discusión.}

La Universidad Metropolitana ha evidenciado a lo largo de su historia, un alto compromiso por asumir la innovación como un elemento clave para el éxito y desempeño institucional, tanto es así que representa uno de sus valores rectores. Al analizar los resultados obtenidos en la investigación, la organización educativa representada a través de las políticas y lineamientos institucionales ha propiciado una gestión capaz de asumir los retos y las demandas que la sociedad de la información y del conocimiento ha impuesto, y se evidencia a través de las instancias y experiencias desarrolladas donde las Tecnologías de la Información y la Comunicación han representado un factor clave, no sólo para los procesos de enseñanza y aprendizaje, sino también para lo relacionado a los procesos académico administrativos que se llevan a cabo en la Universidad; apuntando siempre a visionarse como una institución de vanguardia.

Para los distintos actores educativos objetos de estudio de la presente investigación, surgen un conjunto de elementos que favorecen la gestión de la innovación y que son asumidos como parte de su cultura organizacional, al formar parte de una comunidad como la Unimet; partiendo de que los procesos educativos están centrados en el aprender a aprender, y en el aprendizaje permanente, y el papel del docente es fundamental porque es quien tiene que organizar las nuevas herramientas tecnológicas y adaptarlas a los distintos escenarios educativos diseñando estrategias que favorezcan aprendizajes significativos y gestionando los procesos de enseñanza y aprendizaje; pero también contamos ahora con un nuevo alumno-usuario en el que se acentúa su implicación activa en el proceso de aprendizaje; la atención a las destrezas emocionales e intelectuales a distintos niveles; la preparación de los jóvenes para 
asumir responsabilidades en un mundo en rápido y constante cambio, y la flexibilidad de los estudiantes para entrar en un mundo laboral que demandará formación a lo largo de toda la vida.

Ahora bien, ¿Qué obtuvimos de los sujetos participantes encuestados?; el análisis lo desarrollamos de manera comparativa entre las instancias de acción de la Universidad, y entre los mismos actores educativos como una manera de conocer cómo ellos perciben la gestión de la organización en cuanto a sus procesos de reorganización institucional cuando incorporan las TIC, y si existe realmente o no coherencia entre las distintas instancias.

Los resultados nos han permitido evidenciar que en la universidad los sujetos participantes de la investigación consultados tienen percepciones que no siempre están encaminadas hacia un mismo punto de llegada; en algunos casos opinan que no se está realizando realmente una gestión de la innovación, ello se ha traducido en la sensación de:

Niveles de desconocimiento de los procesos de gestión institucional, por parte de algunos actores educativos.

Deficiencia en la formación y capacitación apoyada en TIC de toda la planta profesoral, por tanto eso imposibilita la continuidad de prácticas educativas innovadoras a lo largo de la formación profesional de los estudiantes.

El problema de la actualización y dotación de la infraestructura tecnológica, en ocasiones se percibe como deficiente; sin embargo es importante destacar en este aspecto lo que implica para la Universidad mantenerse actualizado considerando la situación financiera - política - social actual del país.
Ello nos ha permitido destacar la importancia de la gestión de la innovación orientada por proyectos de innovación que en un marco de gestión institucional permita facilitar la experiencia a los actores que las liderizan, asegurar los procesos de supervisión y evaluación permanente para asegurar que las necesidades están siendo atendidas y los objetivos están orientando las acciones realizadas y asegurar la difusión, ampliación y continuidad de la experiencia de innovación si ella ha sido exitosa y se requiere que perdure en el tiempo. Todo ello vinculado a los planes estratégicos de la institución y de los planes de desarrollo de la nación.

La Unimet, ha venido planificando sus procesos organizando sus recursos (humanos, tecnológicos y económicos) para permitir y facilitar la innovación en la producción y difusión del conocimiento y en los procesos de enseñanza y aprendizaje para probar de manera permanente nuevas alternativas educativas en consonancia con la oferta tecnológica y las demandas sociales, sin embargo aún se deben mejorar algunos procesos para optimizar lo académico y administrativo; y aunque se evidencian experiencias interesantes, con un alto valor educativo e innovadoras, resulta necesario consolidar el trabajo conjunto entre las distintas instancias, de modo que se fortalezca la visión institucional; y sobre todo no haya saturación de esfuerzos de los actores, quienes en muchos casos desconocen lo que hacen sus pares (aspecto que se evidencia en los análisis de discurso llevados a cabo en el apartado de presentación de resultados). 


\section{Referencias Bibliográficas.}

Baptista, L., Fernández, C. \& Hernández, R. (2003). Metodología de la Investigación. Colombia: Editorial Mc Graw Hill.

Barroso, J. (2007). La alfabetización tecnológica CTS. En J. Cabero (Coord.). Nuevas Tecnologías Aplicadas a la Educación. (pp.91-104).Madrid: McGraw-Hill.

Cabero, J. (2005). Las TICs y las Universidades: retos, posibilidades y preocupaciones. Revista de la educación superior, 34 (3), 77-100.

Cabero, J. (2006). Las Nuevas Tecnologías en Sociedad de la Información. En J. Cabero (Coord.). Nuevas Tecnologías Aplicadas a la Educación. Madrid: McGraw-Hill.

Cabero, J., Castaño, C., Cebreiro, B., Gisbert, M., Martínez, F., Morales. J., Prendes, M., Romero, R. \& Salinas, J. (2002). Las nuevas tecnologías en la actividad universitaria. Pixel-Bit. Revista de Medios y Educación, 20,81-100.

Cabero, J. \& Llorente, M. (2006). La rosa de los vientos. Dominios tecnológicos de las TIC por los estudiantes. España: Grupo de Investigación Didáctica. Universidad de Sevilla/Autores.

Camperos. M. (2012). El Proyecto de Evaluación e Investigación evaluativa, sus componentes básicos. Caracas: Fanarte, C.A.

Castaño, C. (Coord.) (2009). Web 2.0: el uso de la Web en la Sociedad del Conocimiento. Investigación e implicaciones Educativa. Caracas, Venezuela: Universidad Metropolitana.

Castell, M. (2002). La dimensión cultural de Internet. Recuperado de http:// www.uoc.edu/culturaxxi/esp/articles/ castells0502/castells0502.html

Delors, J. (1996). La Educación encierra un tesoro. Madrid, España: Santillana.
De Pablos Pons, J. (2012). El cambio metodológico en el espacio europeo de educación superior y el papel de las tecnologías de la información y la comunicación. RIED. Revista Iberoamericana de Educación a Distancia, 10 (2), 15-44. doi: http://dx.doi.org/10.5944/ ried.2.10.992

Díaz, F. (2007). La innovación en la enseñanza soportada en TIC. Una mirada al futuro desde las condiciones actuales. Organización de Estados Iberoamericanos. Recuperado de http://www.oei.es/tic/ santillana/Barriga.pdf

Díaz, R. (2006). Análisis de las relaciones sentido-contexto en la experiencia del «Taller de Educación e Informática» (Tesis doctoral inédita). Universidad Nacional Experimental Simón Rodríguez. Venezuela.

Escudero, J. (2003). Equidad y Calidad: una buena educación para todos. XIX encuentro ADEME, Priego de Córdoba: CEP de Priego- Montilla.

Esteve, F. (2009). Bolonia y las TIC: de la docencia 1.0 al aprendizaje 2.0. La cuestión universitaria, 5, 59-68.

García, L. (2012). Sociedad del Conocimiento y Educación. Madrid: UNED. Recuperado de http://issuu.com/alfonso.df/ docs/sociedad_del_conocimiento _y_educaci_n/1?e=6493193/2609557

García, R. (2001). La Universidad del Siglo $X X I$. Documento presentación en el IX Congreso Iberoamericano de Universidades. México DF: México.

Glaser, B. \& Strauss, A. (1967). The discovery of grounded theory: strategies for qualitative research. New York: Aldine de Gruyter.

Harvey, I. (2010). Desarrollo de una propuesta de gestión de la innovación en la práctica educativa, apoyada en las TIC para 
la Escuela de Educación de la UCV. Tesina para optar al Diploma de Estudios Avanzados (DEA). Universidad de Sevilla. España.

Harvey, I. (2014). Evaluación de un modelo de Gestión de Innovación en la práctica educativa apoyada en las TIC. Estudio de caso: UNIMET (Tesis doctoral inédita). Universidad de Sevilla. Sevilla.

La Guardia, J. (2004). La Gestión de la información y del conocimiento. UCV. Trabajo no publicado.

Morales, V., Medina, E. \& Alvarez, N. (2003). La Educación Superior en Venezuela. Caracas: IESALC-UNESCO.

Morín, E. (2000). Los sietes saberes de la educación del futuro. Caracas: Ediciones Faces/UCV y UNESCO-IESALC.

Navas, E. (2007). La creación de un repositorio de objetos de aprendizaje y su implantación en la Universidad Metropolitana, caso de estudio (Tesis doctoral inédita). Universidad de Sevilla. Sevilla.

Organización Internacional del Trabajo (1998). La educación permanente en el siglo XXI: nuevas funciones para el personal de educación. Informe para el debate de la reunión paritaria sobre la educación permanente en el siglo XXI: nuevas funciones para el personal de educación. Ginebra: Autor.

Rodríguez, E. (2012). Reforma de la educación superior en América Latina. Algunas reflexiones sobre calidad de la enseñanza universitaria, formación del profesorado y las reformas institucionales actuales a partir del análisis del debate en la universidad uruguaya. Unipluriversidad, 6 (2), 85-98.

Rodríguez, M. (2009). Uso didáctico de los Wikis. Caracas, Venezuela: Universidad Metropolitana.
Salinas, J. (2004). Innovación docente y uso de TIC en la enseñanza universitaria. Revista Universidad y Sociedad del Conocimiento, 1 (1), 1-16. Recuperado de http:// www.uoc.edu/rusc/dt/esp/salinas 1104.pdf

Sandín, M. (2003). Investigación cualitativa en educación: fundamentos y tradiciones. Madrid: McGrawHill.

Silvio, J. (2000). La virtualización de la universidad. ¿Cómo podemos transformar la educación superior con la tecnología?. Caracas: IESALC/UNESCO.

UNESCO (2005). Hacia las sociedades del conocimiento. París: UNESCO.

Fecha de recepción: 22-02-2015

Fecha de evaluación: 14-03-2015

Fecha de aceptación: 31-03-2015 